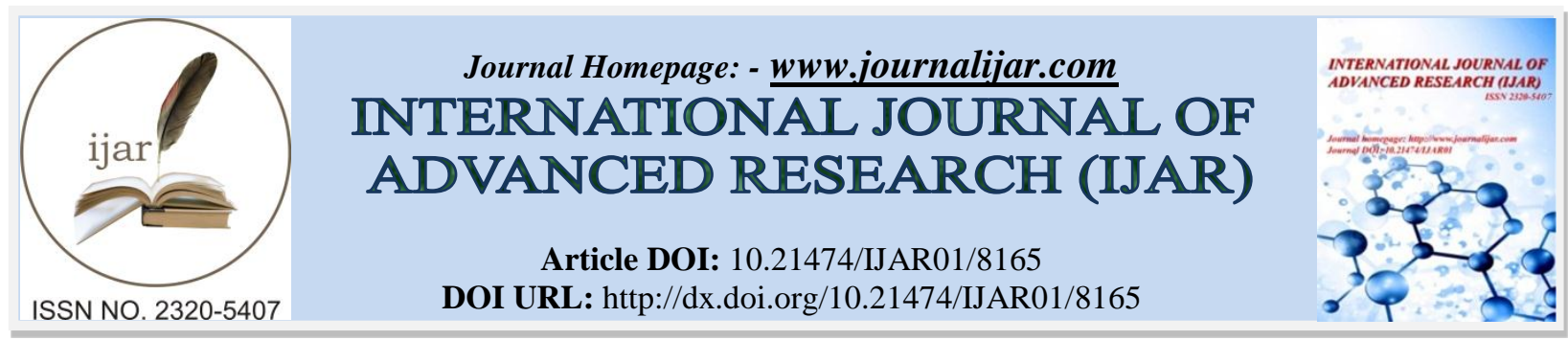

RESEARCH ARTICLE

\title{
COMPARATIVE EVALUATION OF SIALOGRAPHY AND ULTRASONOGRAPHY IN THE DETECTION OF SALIVARY GLAND PATHOLOGIES - AN INVIVO STUDY.
}

\author{
Jiss Mary G ${ }^{1}$, Admaja K Nair ${ }^{2}$, Giju Baby George ${ }^{3}$, Sindhuja Soman ${ }^{4}$, Blesson Abraham Jose ${ }^{5}$. \\ 1. Senior lecturer (OMR) Annoor Dental College. \\ 2. Senior lecturer (OMR),Govt Dental College,Kottayam. \\ 3. Principal and HOD,Annoor Dental College. \\ 4. Post graduate student (Oral Pathology). \\ 5. Post graduate student(OMFS).
}

\section{Manuscript Info}

\section{Manuscript History}

Received: 08 October 2018

Final Accepted: 10 November 2018

Published: December 2018

Keywords:

Sialography,Ultrasonography,Salivary gland diseases

\section{Abstract}

Sialography is one of the oldest imaging procedures and a present day envisage which includes the injection of a radio-opaque media to demonstrate ductal tree of the major salivary glands. A broad spectrum of pathological conditions can affect our salivary glands paramounting to systemic diseases \& this characterizes that salivary gland examination is an important part of oral cavity evaluation.

Significance:In present scenario we have many advanced sophisticated imaging modality. But sialography remains a cost effective specialized imaging technique, if we as oral physicians become competent enough to perform this routinely,it will be an added tool for diagnosis.

Materials and Method : 15 Patients with various symptoms associated with salivary gland pathologies who reported to our institution during the year 2016 - 2017 were subjected to routine radiographic examination and sialography performed in an out-patient basis and ultrasound was done for the same.

Results:Among 15 patients who had symptoms associated with salivary gland pathologies, 6 sialograms were normal.4 had sialolith ,2 had sialectasis, 1 each had benign tumor,secondary sjogrens syndrome and stricture.All these were found in both sialography and ultrasound except for one uncalcified sialolith which was only detected by sialography not in ultrasonography.

Copy Right, IJAR, 2018,. All rights reserved.

\section{Introduction:-}

Sialography or radiosialography is the radiographic visualization of the salivary gland following retrograde instillation of contrast material into the ducts Sialography is one of the oldest imaging procedures and was first mentioned by Carpy in 1902 using mercury as a contrast agent.

Salivary gland disorders range from developmental, inflammatory and immune-related to neoplastic. Treatment primarily depends on diagnosis, which is complemented by the investigations. Conventional sialography remains a useful technique for the investigation of patients presenting with obstructive symptoms of parotid and

Corresponding Author:-Jiss Mary G.

Address:-senior lecturer (OMR) Annoor Dental College. 
submandibular gland evaluating intrinsic and acquired abnormalities of the ductal system because it provides clear visualization of the branching ducts and acinar ends. Sialography can also be used as a therapeutic aid in obstructive salivary gland disorders as it helps in the dislodgement of the small calculi and mucous plugs within the salivary gland ducts which cannot be identified on routine radiographs. Further, sialography can be used in cases of recurrent infections as it helps in salivary gland lavage.s

Detailed imaging of the diseased salivary glands is necessary to diagnose, stage the disease and plan the treatment. Conventional radiography of the salivary glands is a widely accepted technique to detect the calcification within the glands and know the presence of metastasis. But radiographs are not useful to know the extent of destructive and invasive lesions.

Sialography is a radiographic imaging of major salivary glands by introducing a radiopaque contrast medium into the ductal system which is an excellent modality for demonstrating ductal anatomy, presence of obstructions, strictures and are suitable for interventional sialographic procedures.

\section{Materials And Method:-}

15 Patients were reported to Department of Oral Medicine and Radiology ,during the year 2016 - 2017 with symptoms associated with salvary gland disorders, were subjected to routine radiographic examination and sialography was performed and followed by ultrasonography.

We stimulated salivary secretion with citrus fruit as secretogogue and ductal orifice were identified for the gland. With varying sizes of lacrimal dilators, dilated the ductal lumen and cannulated with sialographic cannula of needle size 22 gauge and retrograde instillation of dye done using a $2 \mathrm{ml}$ syringe. We used Iohexol as contrast media which contain $300 \mathrm{meq}$ iodine, $1.25 \mathrm{mg}$ tromethamine, and $0.1 \mathrm{mg}$ calcium edetate and injected contrast media via simple injection technique. (figure 3) Before the procedure, we used intradermal injection of iohexol to see whether any hypersensitivity reaction developing. After that same patients underwent ultrasound examination, and a case - series study was performed in comparison of sialography and ultrasound.

With the sialography we performed we could be able to diagnose a benign tumor, secondary sjogrens syndrome,sialectasis,stricture and sialolith.But in ultrasound we couldn't detect uncalcified silaolith.

\section{Results:-}

Eventhough sialography is one of the oldest imaging technique, with our study we were able to diagnose six conditions associated with salivary gand pathologies in sialography including a benign tumour, secondary sjogrens syndrome, sialectasis ,stricture and sialolith.

\section{Case 1:-}

A 35 year old lady came to our department with chief complaint of dry-mouth and joint pain.Her medical history revealed,positive for RA factor.In sialography a cherry blossom appearance obtained and a mixed hypoechoic and anechoic areas was noted in ultra sound.On the basis of history,examination and investigations, we diagnosed it as a case of secondary sjogrens syndrome.

\section{Case 2:-}

A 60 year old male patient reported with a massive swelling in right side of pre-auricular region and jaw since 2 years with progressive dryness of mouth.In sialography we got a ball in hand appearance and in ultrasound hypoechoic area was obtained and on the basis of this diagnosis of benign tumor of parotid gland and later patient was subjected for parotidectomy and histopathology revealed as pleomorphic adenoma.

\section{CASE 3:-}

A constricted ductal pattern was noted in a patient presented with dry mouth and pain in left submandibular region suggestive of stricture.

\section{CASE 4 and 5:-}

Dilated duct was obtained in 2 patients who had dry-mouth and pain in right sub-mandibular region in sialography and hypeoechoic area in ultrasonography suggestive of sialectasis. 


\section{Case 6,7,8,9:-}

We had 4 cases of sialolith among them one was uncalcified sialolith which was shown only in sialography as a filling defect.No acoustic shadow of the uncalcified sialolith was there in ultrasound.But calcified sialolith was shown in both ultrasound and sialography. (figure 1 and 2)

Among 15 patients we evaluated $40 \%$ sialogram were normal where as $26 \%$ patients were diagnosed with sialolith, $13 \%$ patients were sialectasis, $6 \%$ patients each had benign tumour,secondary sjogrens syndrome and stricture. Later patients with abnormal sialographic appearances were subjected to conventional ultrasonography.Among 26\% patients,sialolith shown as strong hyperechoic lines.No acoustic shadow of uncalcified sialolith was found in ultrasonography.

\section{Discussion:-}

The injection of radiopaque media to supplement a clinical opinion has long been regarded as a valuable aid to diagnosis. Detailed imaging of the diseased salivary glands is necessary to diagnose, stage the disease and plan the treatment. Conventional radiography of the salivary glands is a widely accepted technique to detect pathologies within the gland. But radiographs are not useful to know the extent of destructive and invasive lesions.

Sialography is an important diagnostic procedure in the evaluation of salivary calculi, sialectasis, strictures, fistulae, and tumors. Based on the various sialographic appearances on the radiographs, the salivary gland disorders can be identified.

Sialography is used mainly for diagnostic purposes, but also plays an important role as a therapeutic aid in the treatment of patients suffering from obstructive sialadenitis. ${ }^{i}$ The treatment seems to be effective as it helps clear the mucous plugs and cells in case of recurrent infections.

Normal sialogram of a salivary gland gives a leafless tree pattern.Sjogrens syndrome shows cherry blossom appearance,space occupying lesion manifest as ball in hand appearance.Dilated ductal pattern can be seen in sialectasis and sausage linked pattern will be seen in sialadenitis.But uncalcified, radiolucent sialolith will be seen as filling defect in sialography.

Various types other than conventional sialography includes sialography (with or without digital subtraction),CT sialography (ultrafast technique) and MR sialography.

Sialography with water soluble contrast media material usually is the only means of ruling out salivary calculi.According to Levy and co-workers (1962), these calculi are radiolucent in 20\% of cases,Rubin and Holt (1957) reported that radiolucent stones are found in $20 \%$ submandibular gland cases and $40 \%$ parotid gland cases.Sialography can be used to detect radiolucent calculi.Any delay in evacuation of the contrast media from within the main duct or branches may indicate obstruction by a radiolucent stone.Large radioopaque stones may obstruct the main duct completely. The interface between the stone and contrast medium is characterized by a crescent shaped defect.In most cases contrast medium bypass the stone which causes a filling defect of varying size and shape.

MR sialography is a recent advancement with non-invasive 3D imaging technique. It does not require cannulation of the duct nor does it use a contrast media. It is neither painful nor does it require ionizing radiation.But it has some limitations.According to Mac Kalinowski in 2002 it has low spatial resolution,inferior in diagnosing chronic inflammatory condition.In cases of suspected ductal obstruction, additional thin cross sectional images are indicated.

Ultrasound allows assessment of lesions of the gland parenchyma and intra and extra-glandular duct ectasia, periglandular structures and salivary stones.But it has less application in head and neck region because of difficulty in acoustic shadowing in head and neck region due to the superimposistion of bones and muscles.There is difficulty in localization and interpretation of ultrasound image.Inavailabilty of an intraoral probe is one disadvantage.Also uncalcified sialolith and small sialolith less than $5 \mathrm{~mm}$ cannot be detected in ultrasound ${ }^{\mathrm{ii}}$. All these make conventional sialograhy a better tool for oral physician to aid in diagnosis.Even though it is technique sensitive, if we can combine this with our technical expertise, it can be used widely to detect salivary gland pathologies. 
Song GG etal conducted a meta-analysis in 2014 demonstrates that the diagnostic accuracy of salivary US is comparable with sialography in SS patients.

In our present study,it was found that ultrasound was not able to detect uncalcified sialolith but was only detected in conventional sialography.In a study conducted by Kalka et al, it is possible to achieve diagnostic accuracy with contrast sialography by achieving expertise in the field.

According to Dehghani et al in 1994 proper doses of the iodinated contrast media had no adverse effect on injection.

O'BRIEN and BILLER, 1996 proposed that sialography is an economical diagnostic procedure which provides an accurate view of salivary ducts within and outside of the parotid and mandibular salivary glands.

In our present study also, we found that sialography, eventhough one of the oldest imaging technique, it is very much useful in detecting salivary calculi especially radiolucent stones.

\section{Conclusion:-}

According to revised guidelines of ethical rules 2014,primary objective of every dentist is welfare of patient.So in this era of cry for human rights, as oral physician our ultimate motto should be the same. We have a responsibility to adhere to code of ethics by choosing best and cost effective way for patient care.Role of sialography cant be kept aside.In this era of technological invasion if we combine this type of procedure with technical and practical competency,we can use it wisely to detect salivary gland pathologies. A mere break in our internal notion regarding the technique sensitivity is needed, if so it will definitely be an added tool for oral physician.
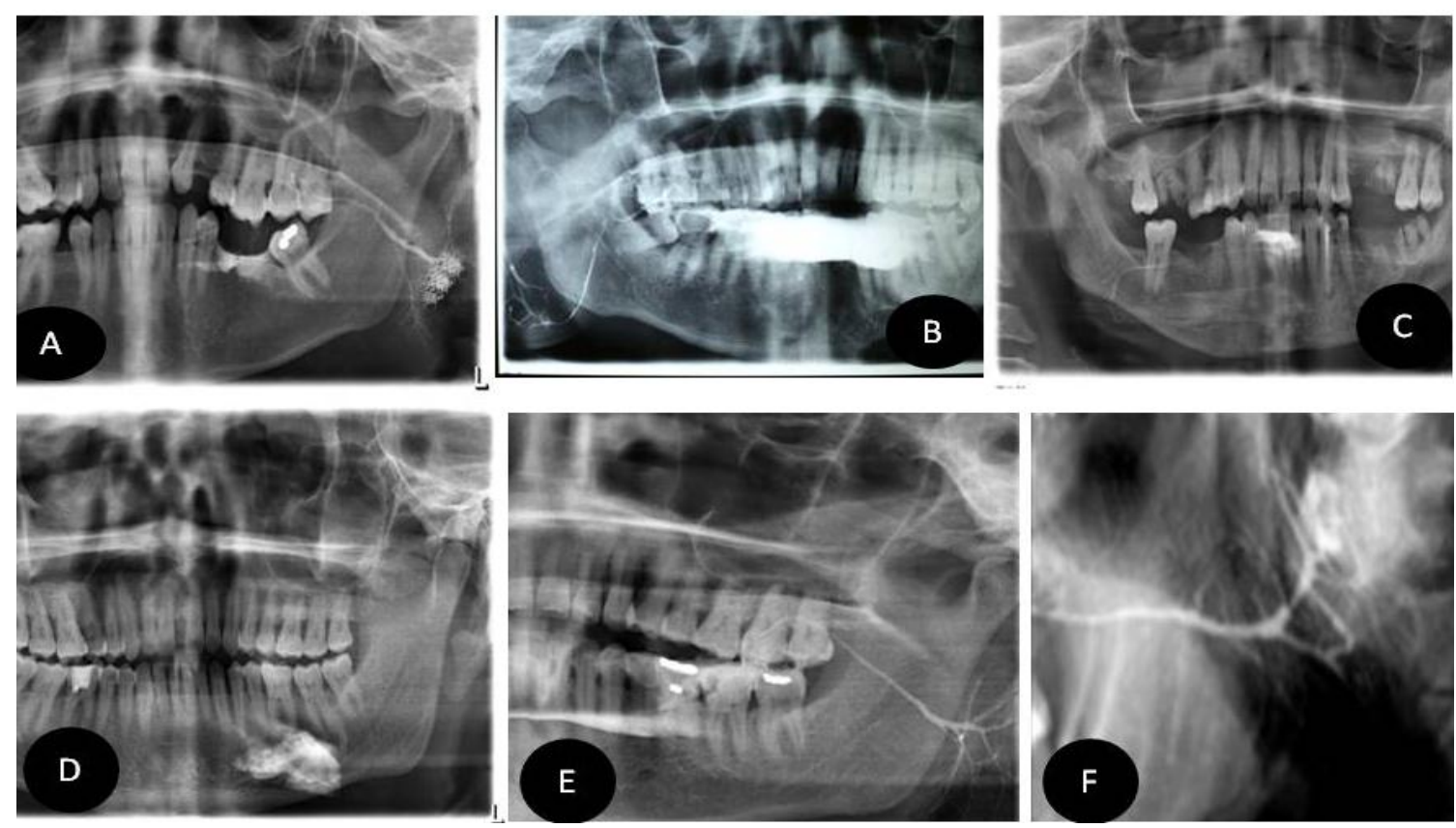

Fig 1:-Cherry blossom appearance as in case 1 . B)Ball in hand appearance as in case 2. C) Dilated duct in case 4 and 5 D)Constricted duct in case 3 E) Calcified sialolith in case 6,7,8 F)Uncalcified sialolith in case 9. 

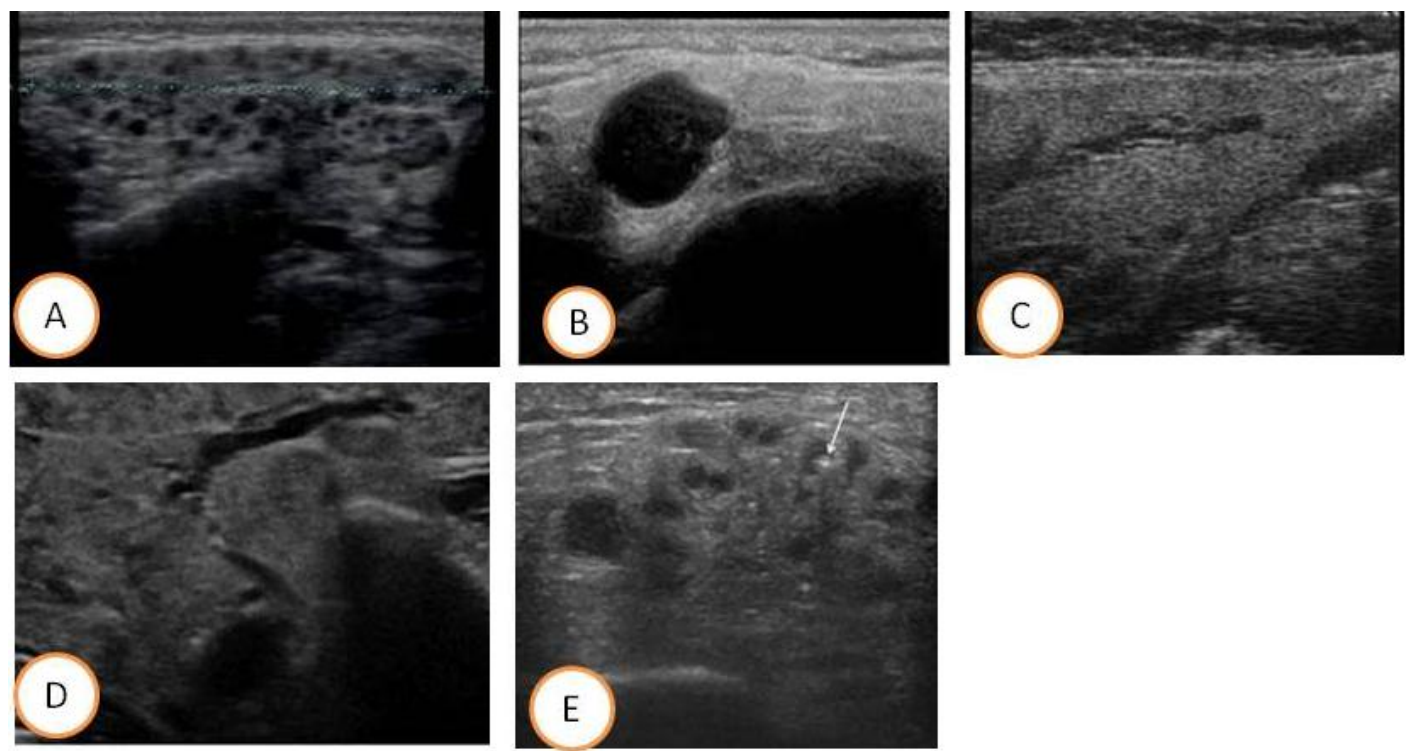

Fig 2:-Mixed hypoechoic area and anechoic area as in case 1 B)Hypoechoic area as in case 2 C)Constricted hypoechoic area as in case 3.D) Dilated hypoechoic area as in case 4 and 5.E)Hyperechoic area suggestive of calcified sialolith

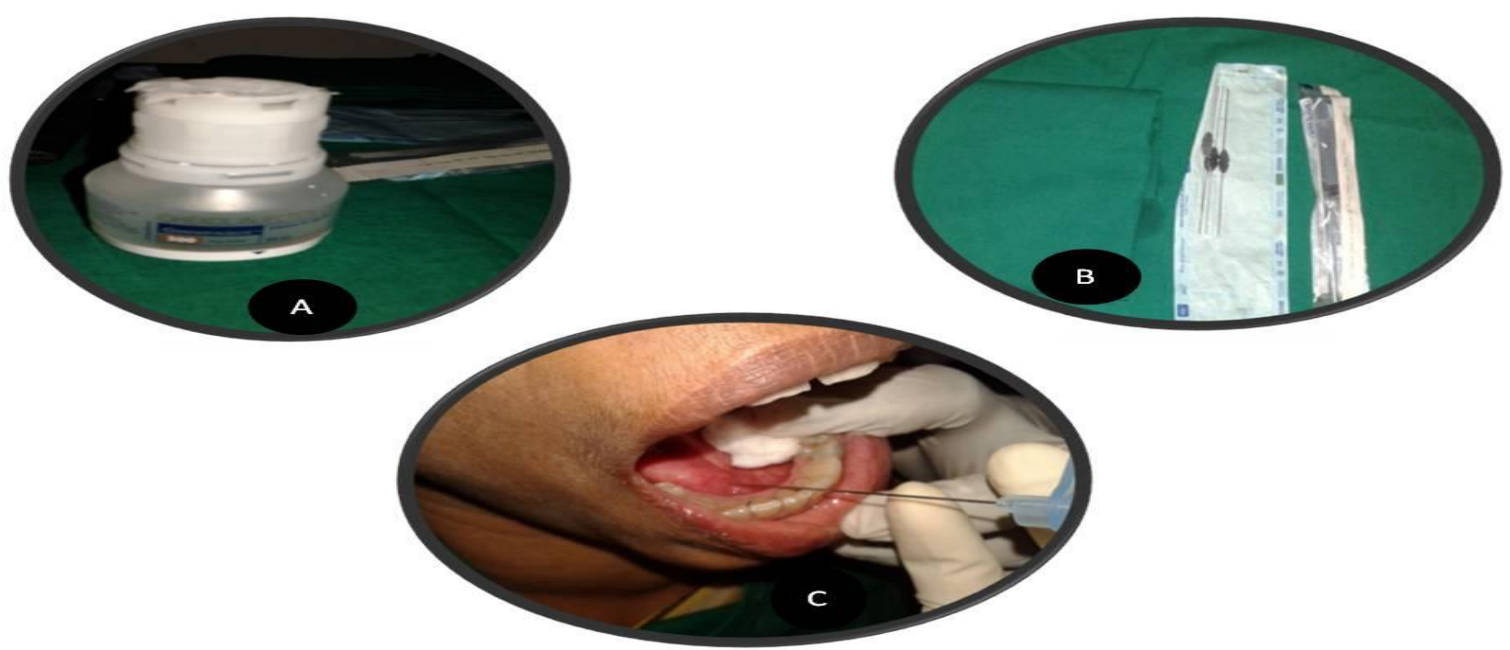

Fig 3:-Iohexol contrast media. B) Lacrimal dilators . C) Cannulation of salivary duct

\section{References:-}

1. Greenberg M S, Glick M. Burket's Oral Medicine, 10th ed. Elsevier publication; 2003. p. 238-9.

2. Malik N. Textbook of Oral and Maxillofacial Surgery.1st ed, jaypee brothers publication, 2002:493-6.

3. Williams MD, Moody AB, Newlands CA, Howlett DC. Gadolinium an alterative contrast agent for sialography in patients with iodine sensitivity. Int J Oral Maxillofac Surg 2003;32:651-2.

4. Malik N. Textbook of Oral and Maxillofacial Surgery.1st ed, jaypee brothers publication, 2002:493-6.

5. Whaites E. Essentials of Dental Radiography and Radiology. Churchill Livingstone; 1992. p. 325-34.

6. Hasson O. Sialoendoscopy and Sialography: Strategies for assessment and treatment of salivary gland obstructions. J Oral Maxillofac Surg

7. 2007;65:300-4.

8. Chitre VV, Premchandra DJ. Recurrent parotitis. Arch Dis Child 1997;77:359-63. 
9. Hupp JR, Ellis E, Tucker MR (2008). Contemporary oral and maxillofacial surgery (5th ed.). St. Louis, Mo.: Mosby Elsevier. pp. 402-405

10. Levy DM, Remine WH, Devine KD. Salivary gland calculi. JAMA 1962;181:1115-9. 68

11. Zbaren P, Ducommun JC. Diagnosis of salivary gland disease using ultrasound and sialography: A comparison. Clin Otolaryngol Allied Sci

12. 2008;14:189-97.

13. Shojaku H, Shojaku H, Shimizu M, Seto H, Watanabe Y. MR sialographic evaluation of sialoctasia of Stensen's duct: Comparison with X-ray

14. sialography and ultrasonography. Radiat Med 2000;1:143-5.

15. Song GG, Lee YH. Diagnostic accuracies of sialography and salivary ultrasonography in Sjögren's syndrome patients: a meta-analysis. Clin Exp Rheumatol. 2014 Jul-Aug;32(4):516-22.

16. Dehghani, s. N., m. Tadjalli, m. H. Masumzadeh ( 2000a): Sialography of sheep parotid and mandibular salivary glands. Res. Vet. Sci. 68, 3-7.

17. O'BRIEN, R. T., D. S. BILLER (1996): Clinical applications of radiography and ancillary imaging. Vet. Clin. North. Am. Food Animal Practice, 263-275. 